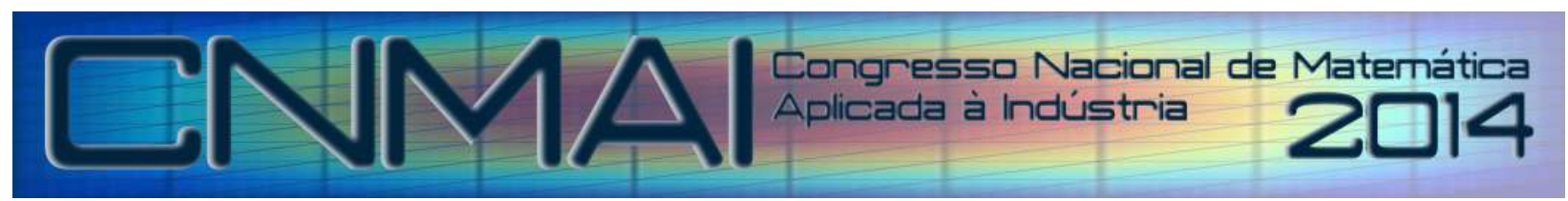

18 a 21 de novembro de 2014, Caldas Novas - Goiás

\title{
ALGORITMO HEURÍSTICO DE RECONFIGURAÇÃO DE SISTEMAS ELÉTRICOS DE DISTRIBUIÇÃO BASEADO NA EQUAÇÃO DE MOVIMENTO DO VAGA-LUME
}

\author{
Lindenberg Isac da Silva, lindenberg.isac@ufabc.edu.br ${ }^{1}$ \\ Edmarcio Antonio Belati, edmarcio.belati@ufabc.edu.br ${ }^{2}$ \\ Ivo Chaves da Silva Junior, ivo.junior@ufjf.edu.br ${ }^{3}$ \\ ${ }^{1,2}$ Universidade Federal do ABC - UFABC - Centro de Engenharia, Modelagem e Ciências Sociais Aplicadas \\ Rua Abolição, s/nº - Vila São Pedro - CEP 09210180 - Santo André - SP - Brasil \\ ${ }^{3}$ Universidade Federal de Juiz de Fora - UFJF - Faculdade de Engenharia - Departamento de Energia Elétrica \\ Rua José Lourenço Kelmer, s/nº - Bairro São Pedro - CEP 36036900 - Juiz de Fora - MG - Brasil
}

\begin{abstract}
Resumo: Um método computacional heurístico baseado na equação de movimento do vaga-lume é apresentado com o intuito de otimizar a distribuição de energia elétrica aos consumidores e minimizar os desperdícios da perda ôhmica por efeito Joule. O algoritmo proposto simula o movimento dos vaga-lumes em busca de presas ou parceiros para acasalamento e, mediante uma discretização, as posições dos insetos no espaço são relacionadas às posições das chaves no sistema elétrico e, com a heurística proposta, reduz o problema do crescimento exponencial da quantidade de combinações de chaveamentos possíveis para reconfigurar o sistema elétrico. Quatro sistemas elétricos foram utilizados nos testes: 16 barras e 16 chaves, 16 barras e 21 chaves, 33 barras e 37 chaves e 70 barras e 74 chaves. $O$ algoritmo, por meio de uma heurística de corte, é capaz de detectar e excluir topologias de rede que transgridam restrições operacionais, tais como a radialidade do sistema e a manutenção do perfil de tensão.
\end{abstract}

Palavras-chave: Sistemas elétricos de potência, Otimização, Reconfiguração

\section{INTRODUÇÃOO}

Sistemas de distribuição de energia elétrica são o estágio final no processo de fornecimento dos geradores aos consumidores individuais. Considerando que estes sistemas operam permanentemente, ou seja, nunca estão sem carga ou desligados, surge a preocupação de reduzir ao máximo os custos desta operação de transporte de energia elétrica da subestação aos consumidores. O desempenho dos sistemas de distribuição diminui consideravelmente conforme aumentam as perdas energéticas devido ao efeito Joule e, consequentemente, redução na magnitude da tensão. Por meio de técnicas de otimização podemos colocar o sistema de distribuição para operar em regime de perdas mínimas, tornando-o mais econômico, além de mais confiável. Pesquisadores estudam vários métodos de otimização para minimizar as perdas ôhmicas, dentre os quais os principais são:
a) utilizar geração distribuída,
b) elevar a tensão do sistema por meio de transformadores,
c) redimensionar os condutores da rede (recabeamento),
d) instalar bancos de capacitores para corrigir o fator de potência das cargas,
e) reconfigurar o sistema por meio da abertura e fechamento de chaves seccionadoras.

A geração distribuída e a reconfiguração são as duas técnicas mais escolhidas atualmente, segundo Dahalan et al. (2013), mas a reconfiguração do sistema elétrico de distribuição é vantajosa por ter custos menores, pois permite reutilizar os equipamentos já existentes no sistema, sem a necessidade de grandes investimentos.

A reconfiguração consiste na abertura e no fechamento de chaves seccionadoras que interligam os dispositivos da rede. Isto faz com que a topologia do sistema seja modificada, remanejando os fluxos de potência de uma linha sobrecarregada para outra menos solicitada naquele instante, o que diminui as perdas de potência ativa. Considerando que uma chave pode assumir apenas dois estados, aberta ou fechada, em um sistema com $n$ chaves temos $2^{n}$ 
possibilidades de reconfiguração para otimizar o sistema elétrico. Dada a característica exponencial do problema combinatorial, um pequeno aumento na quantidade de chaves provoca um grande acréscimo na quantidade de soluções a serem pesquisadas.

Os sistemas com muitas chaves resultam inevitavelmente em uma quantidade enorme de combinações possíveis para a sua reconfiguração, exigindo o emprego de computação científica e técnicas heurísticas que conduzam mais rapidamente à solução do problema. Seria praticamente impossível, devido ao grande tempo gasto, realizar uma reconfiguração de sistema elétrico de distribuição sem utilizar recursos computacionais e mesmo para os engenheiros mais experientes esta possibilidade não é factível.

Neste trabalho, primeiramente expõe-se uma visão geral a respeito da otimização de sistemas elétricos e de alguns trabalhos mais relevantes de autores que empregaram diversas técnicas de reconfiguração. Em seguida, mostra-se a equação de movimento do vaga-lume e seus parâmetros de ajuste. Na seqüência apresenta-se um algoritmo de reconfiguração, baseado na equação de movimento do vaga-lume, com uma heurística adequada a sistemas de distribuição e suas restrições operacionais. Testes computacionais e resultados são mostrados ao fim deste trabalho, comparando os resultados numéricos do método proposto com técnicas de outros autores.

\section{PESQUISAS CORRELATAS EM RECONFIGURAÇÃO}

Há na literatura especializada uma vasta quantidade de trabalhos a respeito da reconfiguração dos sistemas elétricos de distribuição, muitos deles empregando métodos heurísticos, mas nota-se uma carência de aplicação do algoritmo do vaga-lume em sistemas de distribuição. Citamos uma aplicação correlata, mas não igual, deste algoritmo em um sistema de distribuição com geração distribuída e 69 barras, conforme Sulaiman et al. (2012), cujo objetivo é dimensionar e distribuir de forma ótima os geradores. Porém, este propósito é diferente do objetivo deste trabalho que se propõe a reconfigurar as chaves seccionadoras do sistema, embora ambos trabalhos sejam baseados no mesmo princípio do algoritmo do vaga-lume. A seguir, citamos alguns trabalhos notáveis de reconfiguração que empregaram técnicas heurísticas, embora nenhuma utilize o algoritmo do vaga-lume.

No trabalho de Peponis (1995) foi proposto um método heurístico para reconfigurar sistemas de distribuição que consiste em manter a radialidade do sistema compensando o fechamento de uma chave por meio da abertura de outra para que o tamanho da rede não aumente e, consequentemente, não demande mais tempo computacional para a obtenção da solução. A manobra nas chaves é também aproveitada para balancear as cargas entre as fases do sistema. $\mathrm{O}$ método foi aplicado em um sistema de distribuição real de $20 \mathrm{kV}$ na Grécia.

Dois anos depois, Borozan (1997) com base em seus próprios trabalhos de 1995, publicou um método heurístico para reconfigurar sistemas de distribuição trifásicos desbalanceados, visando à diminuição de perdas por efeito Joule, considerando o desequilíbrio entre as cargas. O método foi testado em um sistema de distribuição realístico e nos resultados foi dada ênfase à análise da influência que o desequilíbrio das cargas provoca no resultado da reconfiguração.

Su (2003) publicou um método heurístico chamado de Mixed-Integer Hybrid Differential Evolution (MIHDE) para reduzir as perdas de potência ativa e garantir os níveis de tensão na rede primária de distribuição. Por meio da reconfiguração nas chaves do sistema, a transferência de carga entre os circuitos mantém os níveis de tensão dentro dos parâmetros esperados, considerando que um padrão de carga seja definido para o sistema.

Um algoritmo heurístico apresentado nos trabalhos de Gomes et al. (2005) consiste em iniciar com todas as chaves do sistema fechadas e com base na perda total do sistema, não importando nesta etapa as perdas ôhmicas nos ramos, as chaves são abertas na intenção de eliminar as malhas, sempre que for possível. Na segunda etapa ocorrem as trocas de ramos para reduzir as perdas ativas nos ramos mais críticos. Este algoritmo foi testado usando como referência o sistema de Baran (1989), o de Shirmohammadi (1989), o de McDermott (1999) e também comparado aos trabalhos de Goswami (1992), fornecendo sempre em todos estes testes resultados muito próximos ou iguais ao ótimo global. O método proposto por Gomes et al. (2005) foi verificado por Salume et al. (2006) que o implementou no sistema de 33 barras de Baran (1989) e no sistema de 69 barras de Chiang (1990), mas com a diferença de que Salume et al. (2006) consideraram os sistemas com três fases desequilibradas e, mesmo assim, obtiveram soluções ótimas.

Este panorama nos trabalhos correlatos mostra uma diversidade de métodos para solucionar o problema de reconfiguração, mas aponta também um campo fértil para aplicação do algoritmo do vaga-lume. Embora já existam muitos trabalhos de reconfiguração por meio de enxames de partículas como, por exemplo, algoritmos baseados no comportamento coletivo das formigas de Pereira (2010) e de partículas em geral de Esmin (2005), aparentemente a literatura não está saturada com métodos de reconfiguração por meio do algoritmo do vaga-lume. Isto, dentre outros motivos, serviu de estímulo para o desenvolvimento deste trabalho.

\section{EQUAÇÃO DE MOVIMENTO DO VAGA-LUME}

Yang (2010) propõe, em seu trabalho a respeito de algoritmos heurísticos inspirados no comportamento da natureza, diversos métodos heurísticos para serem aplicados em problemas de otimização em várias áreas do conhecimento. Um destes métodos heurísticos é o algoritmo do vaga-lume, baseado na emissão da luz emitida por este inseto. Os vaga-lumes tendem naturalmente, com base na luminosidade mais forte que avistam, a se concentrarem em regiões nas quais possam ter contato visual com seus possíveis parceiros ou, no caso de presas em potencial, os vagalumes emitem a maior luminosidade possível para atraí-las. Este deslocamento é feito da maneira mais rápida possível 
pelo caminho mais conveniente a eles. Além da distância, a absorção do meio em que a luz se propaga também determina como um vaga-lume enxerga o outro.

O movimento do vaga-lume $i$ em direção ao vaga-lume $j$ com intensidade luminosa mais forte, situados a uma distância $r_{i j}$ um do outro, é dado pela equação de movimento (1):

$$
x_{i} \equiv x_{i}+\beta_{0} e^{-\gamma r_{i j}^{2}}\left(x_{j}-x_{i}\right)+\alpha \varepsilon_{i}
$$

na qual o último termo tem $\alpha$ como um parâmetro de aleatoriedade ajustado pelo usuário e $\varepsilon_{i}$ é outro parâmetro aleatório, mas ajustado automaticamente pelo algoritmo e variando no intervalo probabilístico $[0,1]$ de uma distribuição normal de freqüências (curva gaussiana).

O parâmetro $\beta_{0}$ representa a atratividade entre dois vaga-lumes $i$ e $j$ quando $r_{i j}=0$ e $\gamma$ é um parâmetro de absorção do meio em que a luz se propaga, simulando a ação atmosférica. Para a maioria das aplicações são sugeridos os valores $0 \leq \alpha \leq 1, \beta_{0}=1$ e $0,1<\gamma<10$, segundo os trabalhos originais de Yang (2010). O ambiente no qual os insetos se movimentam pode ser delimitado, influenciando no espaço de busca de soluções do sistema a ser reconfigurado. Podemos delimitar a busca por meio do ajuste $I=\left[x_{\text {min }}, x_{\text {máx }} ; y_{\text {mín }}, y_{\text {máx }}\right]$.

Além dos parâmetros supracitados pertencentes à equação de movimento (1), no algoritmo proposto neste trabalho foi introduzido um parâmetro Z. O objetivo é transformar a terceira componente da coordenada do vaga-lume, a cota $z$, em um número inteiro binário zero ou um, de modo a abrir ou fechar a chave da linha. O valor atribuído ao parâmetro $\mathrm{Z}$ está intimamente ligado ao intervalo $I$, já que a posição do inseto depende de uma função $z=f(x, y)$.

\section{DESCRIÇÃO DO ALGORITMO HEURÍSTICO DE RECONFIGURAÇÃo}

Na heurística empregada no algoritmo de reconfiguração de sistemas de distribuição baseado na equação de movimento do vaga-lume, ARSD-BVL, cada vaga-lume representa uma chave no sistema elétrico. Assim, a população é sempre igual à quantidade de chaves. A posição espacial de cada inseto determina o estado da respectiva chave. Todas as linhas são candidatas ao chaveamento, ou seja, há uma chave manobrável em cada linha.

A função objetivo original apresentada por Yang (2010) possui quatro pontos de máximo e é exponencial. No problema de reconfiguração a função objetivo que é sujeita às restrições operacionais da rede e que deve ser minimizada, representando a perda de potência ativa $(P)$ em $N$ linhas do sistema elétrico, é dada por

$$
f(P)=\sum_{n=1}^{N} R_{n} i_{n}^{2},
$$

com $R$ e $i$ simbolizando, respectivamente, resistência e corrente elétrica, de acordo com Opazo et al. (2008). A equação (2) comparece no ARSD-BVL como função objetivo e determina o posicionamento otimizado dos vaga-lumes, segundo uma ogiva formada pela função quadrática com duas variáveis independentes $(x, y)$ e uma variável dependente $(z)$.

Em um sistema elétrico com $n$ chaves, cada iteração realizada resulta em um vetor com o posicionamento espacial dos $n$ insetos e, após uma discretização destas coordenadas, obtém-se um vetor de estado representando uma possível solução de reconfiguração. Cada vetor de estado criado é concatenado como uma nova linha em uma matriz de estados, ou seja, a quantidade de linhas desta matriz equivale à quantidade de iterações realizadas.

Uma quantidade maior de iterações resulta em uma probabilidade mais alta de encontrarmos uma solução ótima global de reconfiguração. Quantidades menores de iterações, desde que os parâmetros estejam devidamente ajustados, conduzem ao encontro de soluções ótimas locais factíveis e de muito boa qualidade.

Após a obtenção da matriz de estados, são detectados e excluídos os vetores com restrições operacionais, tais como topologias malhadas ou desconexas (ilhamento de barras), bem como os estados em que os limites mínimo e máximo de tensão seriam excedidos. Sob o ponto de vista computacional, ignorar tais vetores de estado significa economizar tempo em não ter que calcular fluxos de potência de estados que não são factíveis. O estabelecimento de limites entre 0,95 p.u. e 1,05 p.u. para a tensão satisfaz a maioria das restrições operacionais dos equipamentos instalados no sistema elétrico, bem como atende as exigências impostas para a tensão de fornecimento em redes de distribuição.

O fluxo de potência nas linhas do sistema elétrico é então calculado computacionalmente por meio do método de Newton-Raphson. Em geral, são suficientes no máximo quatro iterações para a obtenção das variáveis de estado do sistema elétrico. A perda ôhmica em cada linha ligando as barras $k$ e $m$ considerando, respectivamente, $a, g, V, \theta, \varphi$ como a relação do transformador em fase, a condutância, a tensão, o ângulo de fase e o ângulo do transformador defasador, é calculada por meio da equação (3):

$$
P_{\text {perda }}=a^{2} g_{k m}\left(V_{k}^{2}+V_{m}^{2}\right)-2 a V_{k} V_{m} g_{k m} \cos \left(\theta_{k m}+\varphi_{k m}\right) \text {. }
$$

As menores perdas ôhmicas das melhores topologias candidatas à solução são memorizadas com seus respectivos chaveamentos. As chaves abertas destes vetores são então combinadas para uma nova pesquisa em busca do ótimo global. É feita uma segunda verificação em busca de possíveis vetores com restrições operacionais, bem como uma avaliação final da menor perda ôhmica total encontrada, que será a solução do problema de reconfiguração. 
Em uma analogia entre o comportamento coletivo dos vaga-lumes e o sistema elétrico, a corrente elétrica tende a se deslocar da região de maior potencial para a de menor potencial, sempre pelo caminho de menor resistência elétrica. Este percurso é representado fisicamente pelo condutor que une as barras $k$ e $m$ em um sistema elétrico e cuja interdistância é $\mathrm{km}$. A queda de tensão neste condutor, causada pela resistência elétrica do material, pode ser comparada ao parâmetro que provoca atenuação da luz emitida pelo vaga-lume.

\section{ANÁLISE DE SENSIBILIDADE DOS PARÂMETROS}

No menor sistema testado neste trabalho empregamos um intervalo de busca menor, compreendido entre $I=\left[x_{\text {min }}, x_{\text {máx }} ; y_{\text {min }}, y_{\text {máx }}\right]=[0,5 ; 0,5]$, o que faz com que a procura pelas soluções seja agilizada. No entanto, para sistemas com maior quantidade de chaves, um intervalo demasiadamente estreito pode esconder possíveis soluções ótimas locais e até mesmo globais, prejudicando a reconfiguração do sistema elétrico. Para o maior sistema testado, utilizamos o intervalo $I=[0,13 ; 0,13]$, o que resultou na localização da solução ótima global. Intervalos menores resultaram em soluções ótimas locais. Em suma, deve-se expandir o intervalo $I$ de busca conforme a quantidade de chaves no sistema elétrico.

O parâmetro de aleatoriedade permitiu a localização de soluções no menor sistema testado quando ajustado em $\alpha=0,45$ e, no maior sistema testado, $\alpha=0,55$. Verificou-se experimentalmente que as soluções de reconfiguração são encontradas quando $0,45 \leq \alpha \leq 0,55$. Valores maiores deste parâmetro resultam em uma maior diversidade no chaveamento, compensando um aumento na quantidade de chaves dos grandes sistemas e permitindo encontrar ótimos locais. Se este parâmetro for diminuído, a busca de soluções concentra-se em torno de chaveamentos parecidos entre si, o que facilita localizar ótimos globais quando simultaneamente encurtamos $I$. Porém, valores demasiadamente grandes de $\alpha$ em relação à quantidade de chaves prejudica uma busca mais concentrada em determinado espaço em que possivelmente existam soluções ótimas.

O parâmetro de absorção atmosférica da luz dos vaga-lumes foi ajustado em $\gamma=4,2$ para os quatro sistemas testados neste trabalho, de maneira a atribuir um valor universal a $\gamma$, diminuindo o trabalho de ajuste de parâmetros. Valores mais altos de $\gamma$ produzem uma convergência mais lenta dos vaga-lumes em direção ao ponto notável da função objetivo, implicando em vetores de estado com muitas chaves na mesma posição (aberta ou fechada) e, consequentemente, em um aumento indesejável das iterações para encontrar a solução. $\mathrm{O}$ parâmetro $Z=2$ também foi fixado para todos os sistemas testados neste trabalho, de modo a se comportar como um valor universal diante de qualquer quantidade de chaves no sistema elétrico.

\section{TESTES E RESULTADOS}

Quatro sistemas elétricos hipotéticos foram reconfigurados. Na figura 1 tem-se o primeiro sistema de distribuição reconfigurado, com 16 barras, sendo três subestações e as demais barras de carga. A potência de base equivale a 100 MVA. Os dados de linha e de barra encontram-se originalmente nos trabalhos de Civanlar et al. (1988) e posteriormente também nos trabalhos de Pereira (2010). Considerando 16 linhas com uma chave seccionadora em cada, o total de possibilidades de chaveamento é de $2^{16}=65536$.

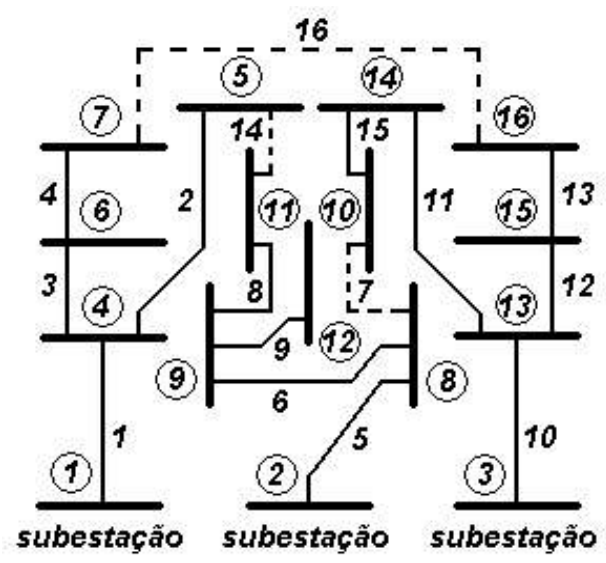

Figura 1. Sistema de distribuição de 16 barras e 16 linhas. Linhas tracejadas representam chave aberta.

Mediante 90 iterações e após a remoção de estados restritos, gerou-se uma matriz de estados com 67 vetores de estado factíveis, cada um representando um possível estado de chaveamento, dentre os quais se encontrou o chaveamento da solução ótima global com topologia radial e as chaves 7, 8 e 16 abertas, resultando em perda ôhmica total de 466,092 kW. 
Na tabela 1 tem-se uma comparação entre os resultados obtidos pelo ARSD-BVL e aqueles publicados por outros autores empregando técnicas diferentes. Nota-se que, com o algoritmo proposto, também é possível encontrar diversas soluções ótimas locais, além da solução ótima global.

Tabela 1. Comparação entre a perda de potência ativa total entre diferentes experimentos no sistema de 16 chaves.

\begin{tabular}{cccc}
\hline Experimento & Método & $\begin{array}{c}\text { Perda ôhmica } \\
\text { total }(\mathrm{kW})\end{array}$ & $\begin{array}{c}\text { Chaves } \\
\text { abertas }\end{array}$ \\
\hline ARSD-BVL & Vaga-lume & 466,092 & $7,8,16$ \\
Gomes & Redes fictícias, otimização contínua & 466,130 & $7,8,16$ \\
Guimarães & Busca Tabu & 466,130 & $7,8,16$ \\
Lorenzeti & Análise de estabilidade de tensão & 466,130 & $7,8,16$ \\
Mantovani & Troca de ramos com fluxo de carga rápido & 466,130 & $7,8,16$ \\
Pereira & Formiga & 466,130 & $7,8,16$ \\
ARSD-BVL & Vaga-lume & 479,256 & $4,7,8$ \\
ARSD-BVL & Vaga-lume & 483,828 & $7,14,16$ \\
Civanlar & Troca de ramos & 483,880 & $7,14,16$ \\
ARSD-BVL & Vaga-lume & 493,109 & $8,15,16$ \\
\hline
\end{tabular}

Pequenas variações na perda ôhmica calculada nos experimentos da tabela 1, para um mesmo chaveamento, são justificadas pela diferença na precisão entre os métodos usados para cálculo do fluxo de potência. Além disso, a precisão numérica dos computadores utilizados pode ser simples (32 bits), dupla (64 bits) ou estendida (80 bits), às vezes influenciando no resultado final de experimentos diferentes.

$\mathrm{Na}$ figura 2 tem-se o segundo sistema de distribuição reconfigurado nos testes, com 16 barras e 21 linhas, hipoteticamente proposto por Abur (1996), sendo uma subestação na barra 1 (referência) e as demais barras de carga. A potência de base equivale a 100 MVA. Considerando cada linha candidata à manobra de sua respectiva chave, o total de possibilidades de chaveamento é de 2.097.152. Com 120 iterações foi possível encontrar uma solução ótima local dentre 83 vetores de estado factíveis, candidatos à solução. Com 260 iterações, o que resultou em 227 vetores de estados factíveis, o ARSD-BVL encontrou a solução ótima global. Nota-se pela quantidade de vetores de estado pesquisados, que o espaço de busca das soluções reduziu bastante em relação ao total de possibilidades de chaveamento apresentado por este sistema.

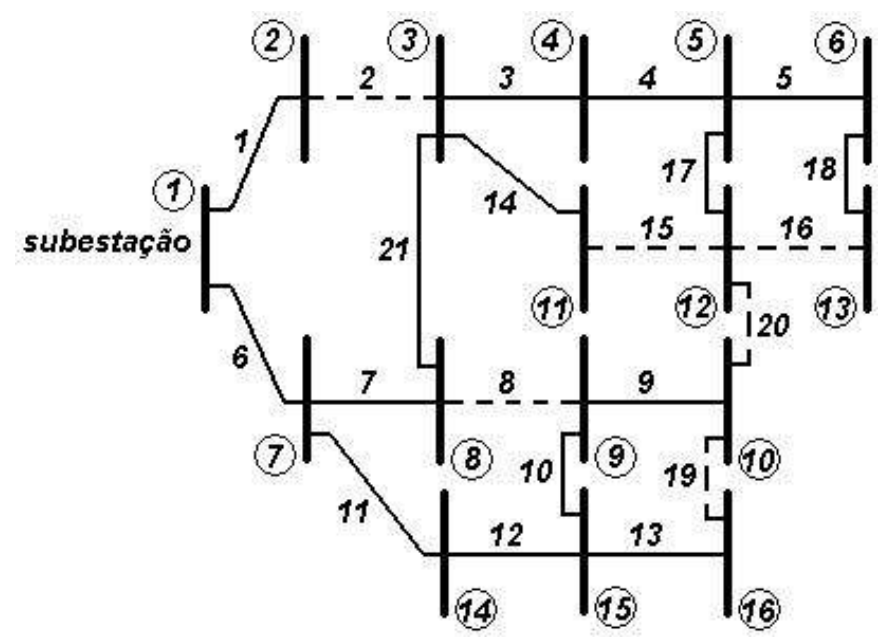

Figura 2. Sistema de distribuição de 16 barras e 21 linhas. Linhas tracejadas representam chave aberta.

Abur (1996) propôs a reconfiguração deste sistema considerando a restrição operacional de tensão $V \geq 0,95 p . u$. e depois a restrição de 0,9 p.u. $\leq V<0,95$ p.u., cujos resultados de reconfiguração aparecem, respectivamente, nas tabelas 2 e 3, a seguir. Nota-se que o ARSD-BVL conseguiu obter, além da solução ótima global, soluções ótimas locais, todas satisfazendo as restrições operacionais impostas.

Tabela 2. Perda de potência ativa total no sistema de 21 chaves com $V \geq 0,95$ p.u..

\begin{tabular}{ccccc}
\hline Experimento & Método & $\begin{array}{c}\text { Perda ôhmica } \\
\text { total (MW) }\end{array}$ & $\begin{array}{c}\text { Tensão mínima } \\
\text { (p.u.) [barra] }\end{array}$ & Chaves abertas \\
\hline ARSD-BVL & Vaga-lume & 9,996 & $0,952[12]$ & $2,8,15,16,19,20$ \\
ARSD-BVL & Vaga-lume & 10,029 & $0,951[12]$ & $2,10,15,16,19,20$ \\
Abur & Programação linear & 10,034 & $0,952[12]$ & $2,8,9,15,16,20$ \\
ARSD-BVL & Vaga-lume & 10,152 & $0,950[13]$ & $2,8,9,15,18,20$ \\
\hline
\end{tabular}


Tabela 3. Perda de potência ativa total no sistema de 21 chaves com 0,9 p.u. $\leq V<0,95$ p.u. .

\begin{tabular}{cccc}
\hline Experimento & $\begin{array}{c}\text { Perda ôhmica } \\
\text { total (MW) }\end{array}$ & $\begin{array}{c}\text { Tensão mínima (p.u.) } \\
\text { [barra] }\end{array}$ & Chaves abertas \\
\hline ARSD-BVL & 9,950 & $0,947[12]$ & $10,15,16,17,19,2120$ \\
ARSD-BVL & 10,426 & $0,946[11]$ & $2,8,14,16,19,20$ \\
ARSD-BVL & 10,489 & $0,944[12]$ & $2,8,16,17,19,20$ \\
ARSD-BVL & 10,587 & $0,946[11]$ & $2,8,14,18,19,20$ \\
Abur & 14,210 & $0,918[13]$ & $10,17,18,19,20,21$ \\
\hline
\end{tabular}

Na figura 3 tem-se o terceiro sistema de distribuição reconfigurado nos testes, com 33 barras e 37 linhas, sendo uma subestação na barra 1 (referência) e as demais barras de carga. A potência de base equivale a 1 MVA e a tensão de base é de 12,66 kV. Os dados de linha e de barra deste sistema encontram-se originalmente nos trabalhos de Baran (1989) e posteriormente também nos trabalhos de Pereira (2010). Considerando 37 chaves, isto é, uma chave seccionadora em cada linha, o total de possibilidades de chaveamento é de $2^{37} \cong 137 \cdot 10^{9}$, tornando bem demorada a resolução computacional por busca exaustiva, pois seria necessário calcular o fluxo de potência e perdas ôhmicas cerca de 137 bilhões de vezes para descobrir o estado de menores perdas ôhmicas.

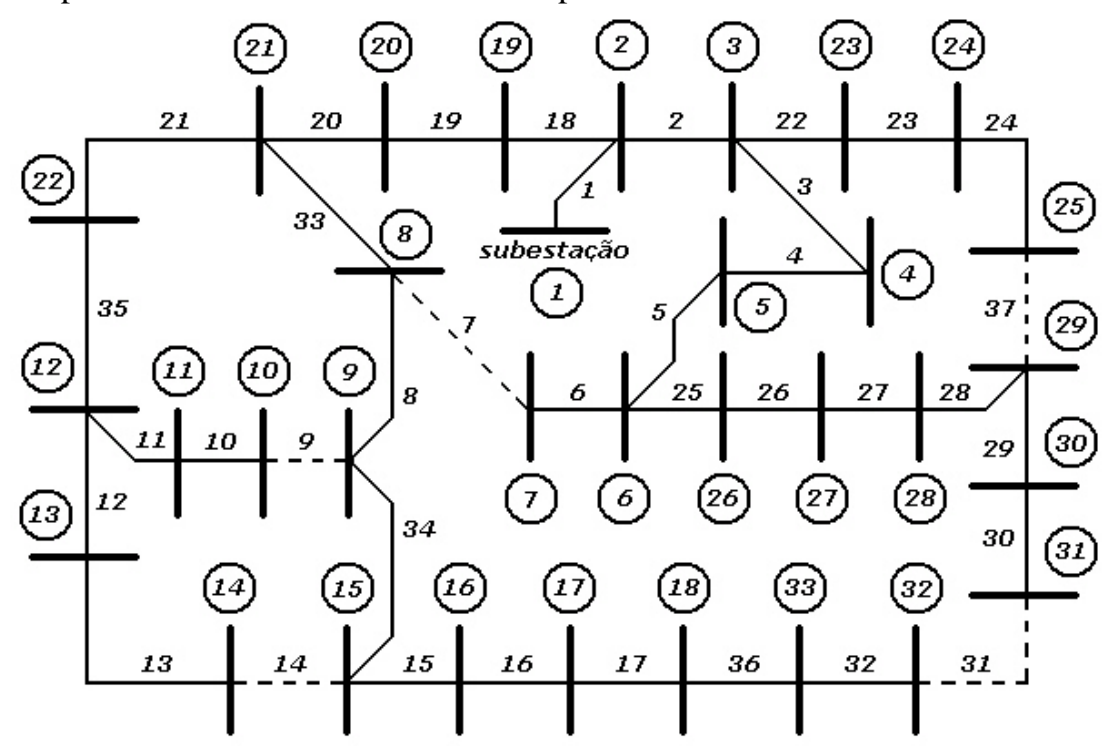

Figura 3. Sistema de distribuição de 33 barras e 37 linhas. Linhas tracejadas representam chave aberta.

Com 280 iterações o ARSD-BVL gerou uma matriz de estados com 274 linhas, encontrando soluções ótimas locais tão boas quanto as de Baran (1989) e Pereira (2010). Com 1350 iterações e uma matriz de estados com 1314 vetores candidatos à solução, foi possível localizar a mesma topologia da solução ótima global encontrada por Gomes (2005), conforme mostrado na tabela 4 .

Tabela 4. Comparação entre a perda de potência ativa total entre diferentes experimentos no sistema de 37 chaves.

\begin{tabular}{ccc}
\hline Experimento & $\begin{array}{c}\text { Perda ôhmica } \\
\text { total }(\mathrm{kW})\end{array}$ & Chaves abertas \\
\hline Gomes & 137 & $7,9,14,32,37$ \\
ARSD-BVL & 139 & $7,9,14,32,37$ \\
ARSD-BVL & 140 & $7,9,14,28,32$ \\
Pereira & 140 & $7,9,14,28,32$ \\
ARSD-BVL & 141 & $7,10,14,28,32$ \\
ARSD-BVL & 142 & $7,9,14,28,36$ \\
ARSD-BVL & 143 & $7,9,14,31,37$ \\
ARSD-BVL & 147 & $7,8,28,32,34$ \\
Baran e Wu & 161 & $7,10,14,27,30$ \\
\hline
\end{tabular}

O quarto e último sistema reconfigurado nos testes tem 70 barras e 74 linhas, sendo uma subestação na barra 1 e as demais barras de carga. A potência de base equivale a 100 MVA e a tensão de base é 12,66 kV. Os dados de barras e de linhas deste sistema encontram-se no trabalho de Chiang (1990). As 74 chaves resultam em uma explosão combinatorial de $2^{74} \cong 1,89 \cdot 10^{22}$ possibilidades de chaveamento para reconfigurar este sistema. Na figura 4 apresenta-se o diagrama unifilar deste sistema elétrico de distribuição. 


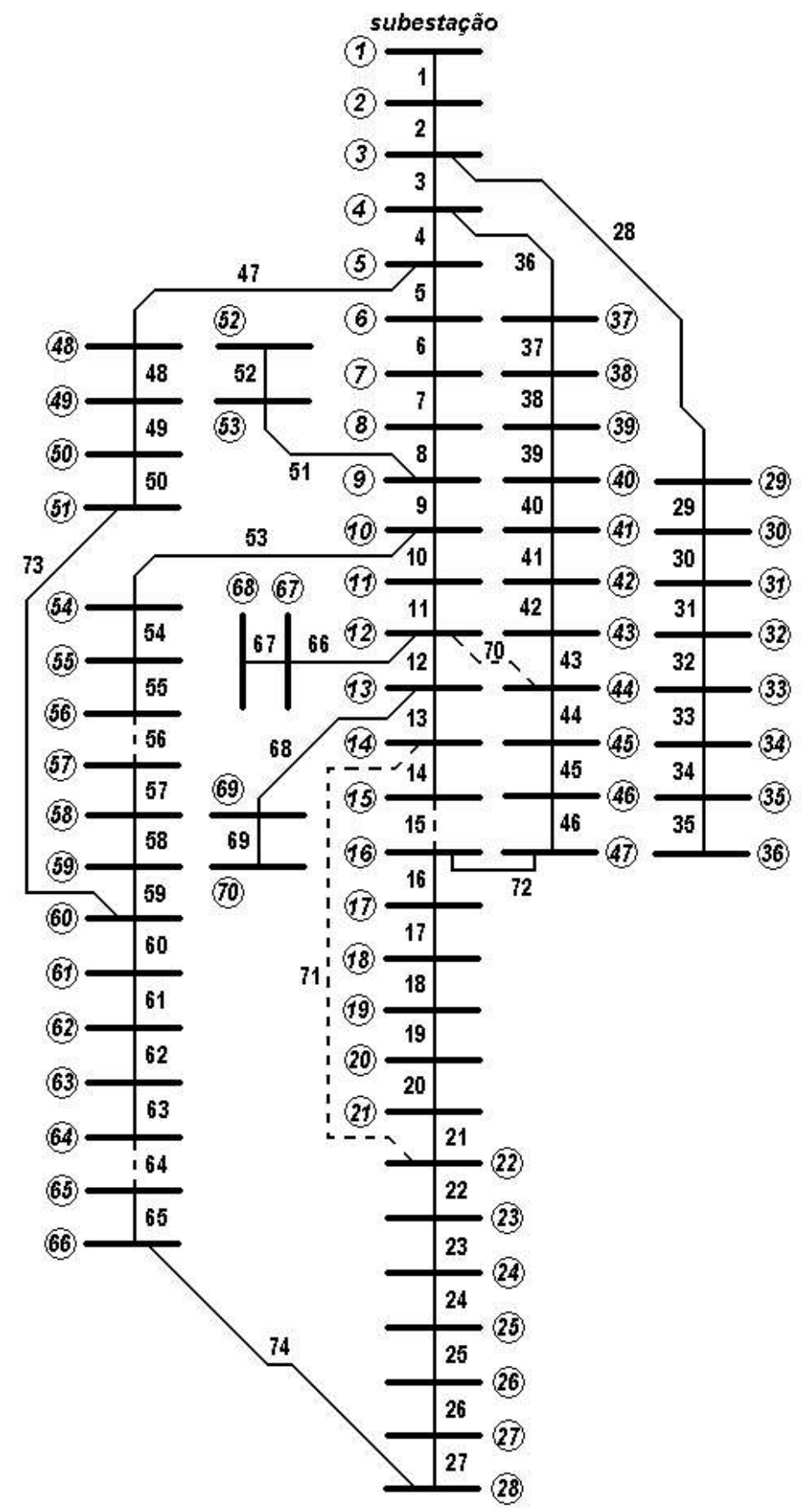

Figura 4. Sistema de distribuição de 70 barras e 74 linhas. Linhas tracejadas representam chave aberta.

Na tabela 5 há uma comparação entre os resultados obtidos pelo ARSD-BVL e dos trabalhos de Guimarães (2005) e Lorenzeti (2004).

Tabela 5. Comparação entre a perda de potência ativa total entre diferentes experimentos no sistema de $\mathbf{7 4}$ chaves.

\begin{tabular}{ccc}
\hline Experimento & $\begin{array}{c}\text { Perda ôhmica } \\
\text { total }(\mathrm{kW})\end{array}$ & Chaves abertas \\
\hline Guimarães & 9,41 & $15,57,62,70,71$ \\
Guimarães & 9,41 & $15,59,62,70,71$ \\
Lorenzeti & 9,42 & $14,58,62,70,71$ \\
ARSD-BVL & 9,50 & $15,56,64,70,71$ \\
ARSD-BVL & 9,51 & $14,56,64,70,71$ \\
ARSD-BVL & 9,52 & $13,56,64,70,71$ \\
ARSD-BVL & 9,86 & $11,14,55,64,71$ \\
\hline
\end{tabular}


A partir de 800 iterações o ARSD-BVL consegue encontrar as soluções ótimas locais que aparecem na tabela 5, melhorando o refinamento da solução conforme se aumenta a quantidade de iterações. Após a reconfiguração do sistema, constata-se tensão mínima de $V_{\text {min }}=0,982$ p.u. nas barras 62, 63 e 64 para a topologia com as chaves 15, 56, 64, 70, 71 abertas, a melhor encontrada pelo ARSD-BVL. Percebe-se que $V_{\text {min }}$, na prática, não viola as restrições operacionais de tensão da rede de distribuição.

\section{FLUXOGRAMA}

A obtenção da solução do fluxo de potência otimizado por meio do ARSD-BVL requer o cumprimento das seguintes etapas computacionais mostradas no fluxograma da figura 5, a seguir:

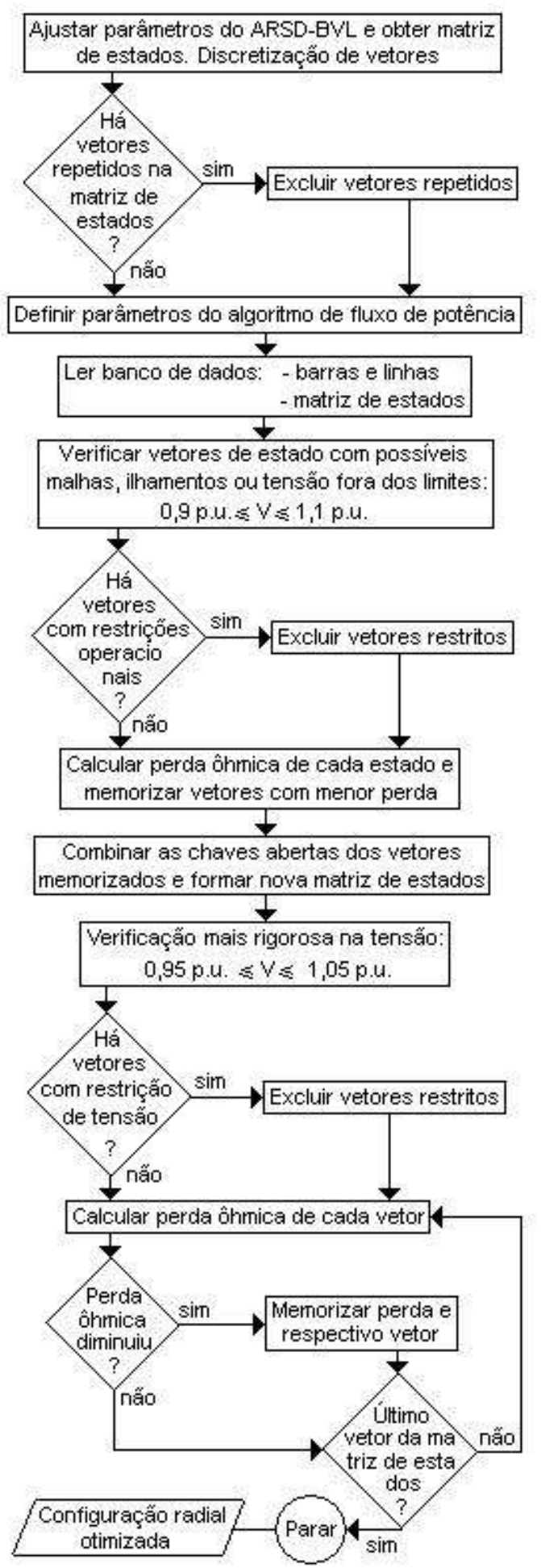

Figura 5. Fluxograma do algoritmo proposto. 


\section{VERIFICAÇÃO DO MÉTODO PROPOSTO}

Vetores de estados randômicos foram gerados por meio do software Matlab, com distribuição normal (gaussiana) e depois com distribuição contínua, ambas no intervalo $I=[0,1]$. Notou-se, conforme tabela 6 , que a criação de vetores de estado nestas condições, na tentativa de encontrar soluções para o problema de reconfiguração, funcionou somente em sistemas com poucas combinações de chaveamento, sendo que em sistemas com grande quantidade de combinações, mesmo gerando milhares de vetores de estados randômicos, não foi possível encontrar um único estado factível.

Tabela 6. Quantidade necessária de vetores de estado para determinação de uma solução (local ou global) para o problema de reconfiguração.

Quantidade de vetores de estado para encontrar ao menos uma solução [tipo de solução]

\begin{tabular}{ccccc}
\hline $\begin{array}{c}\text { Sistema } \\
\text { (chaves) }\end{array}$ & Distribuição Normal & Distribuição Contínua & ARSD-BVL \\
\hline 16 & 10800 [local] & 11070 [local] & 90 [global] \\
& $>10800$ [nenhuma] & $>11070$ [nenhuma] & 120 [local] \\
21 & & & [global] \\
& $>10800$ [nenhuma] & $>11070$ [nenhuma] & 280 [local] \\
37 & $>>1350$ [global] & \\
74 & & & 800 [local] \\
\hline
\end{tabular}

\section{CONCLUSÕES}

O método heurístico proposto aparenta ser promissor no emprego em sistemas de distribuição com maior quantidade de barras, visto que funcionou com êxito em quatro sistemas de distribuição que apresentam restrições operacionais. Foram obtidos resultados iguais e às vezes melhores que os alcançados por outros métodos. Percebeu-se o quão importante é o correto ajuste nos parâmetros do algoritmo, de acordo com as características de cada sistema a ser reconfigurado, sendo este cuidado essencial para o correto funcionamento do método. A continuidade no desenvolvimento do projeto proposto consistirá em uma pesquisa significativa a respeito do algoritmo heurístico do vaga-lume que, conforme as referências consultadas, ainda apresenta uma certa carência de aplicações em problemas de reconfiguração de sistemas elétricos.

\section{AGRADECIMENTOS}

Agradecemos à Coordenação de Aperfeiçoamento de Pessoal de Nível Superior - CAPES, fundação do Ministério da Educação - $M E C$, pelo apoio financeiro prestado.

\section{REFERÊNCIAS}

Abur, Ali. A modified linear programming method for distribution system reconfiguration. Electrical Power \& Energy Systems, v. 18, n. 7, p. 469-474, 1996.

Baran, M. E.; Wu, F. F. Network reconfiguration in distribution systems for loss reduction and load balancing. IEEE Transactions On Power Delivery, v. 4, n. 2, p. 1401-1407, 1989.

Borozan, Vesna; Rajičić, Dragoslav; Ačkovski, Risto. Minimum loss reconfiguration of unbalanced distribution networks. IEEE Transactions On Power Delivery, v. 12, n. 1, p. 435-442, 1997.

Chiang, Hsiao-Dong; Jean-Jumeau, René. Optimal network reconfigurations in distribution systems part 2: solution algorithms and numerical results. IEEE Transactions On Power Delivery, v. 5, n. 3, p. 1568-1574, 1990.

Civanlar, Seyhan et al. Distribution reconfiguration for loss reduction. IEEE Transactions On Power Delivery, v. 3, n. 3, p. 1217-1223, 1988.

Dahalan, Wardiah Mohd et al. The simultaneous application of optimum network reconfiguration and distributed generation sizing using PSO for power loss reduction. Przegląd Elektrotechniczny, v. 89, n. 4, p. 137-141, 2013.

Esmin, Ahmed Ali Abdalla. Estudo de aplicação do algoritmo de otimização por enxame de partícula na resolução de problemas de otimização ligados ao SEP. 2005. Tese (Doutorado em Engenharia Elétrica) - Universidade Federal de Itajubá, Minas Gerais, 2005.

Gomes, Flavio Vanderson et al. A new heuristic reconfiguration algorithm for large distribution system. IEEE Transactions On Power Systems, v. 20, n. 3, p. 1373-1378, 2005.

Goswami, Swapan Kumar; Basu, Sanjoy Kumar. A new algorithm for the reconfiguration of distribution feeders for loss minimization. IEEE Transactions On Power Delivery, v. 7, n. 3, p. 1484-1491, 1992. 
Guimarães, Marcos Antonio do Nascimento. Reconfiguração de sistemas de distribuição de energia elétrica utilizando algoritmos de busca Tabu. 2005. Dissertação (Mestrado em Engenharia Elétrica) - Faculdade de Engenharia Elétrica e Computação da Universidade Estadual de Campinas, Campinas, 2005.

Lorenzeti, J. F. C. Reconfiguração de sistemas de distribuição de energia elétrica para a melhoria das condições de operação com relação à estabilidade de tensão. 2004. Dissertação (Mestrado em Engenharia Elétrica) - Faculdade de Engenharia Elétrica e Computação da Universidade Estadual de Campinas, Campinas, 2004.

Mantovani, José R. S.; Casari, Fernando; Romero, Rubén A. Reconfiguração de sistemas de distribuição radiais utilizando o critério de queda de tensão. Revista SBA Controle \& Automação, v. 11, n. 3, p. 150-159, set./out./nov./dez. 2000.

McDermott, Tom E.; Drezga, Irislav; Broadwater, Robert P. A heuristic nonlinear constructive method for distribution system reconfiguration. IEEE Transactions On Power Systems, v. 14, n. 2, p. 478-483, 1999.

Opazo, Hugo et al. Minimal loss reconfiguration considering random load: applications to real networks. Ingeniare Revista Chilena de Ingeniería, v. 16, n. 1, p. 264-272, 2008.

Peponis, G. J.; Papadopoulos, Michael P. Reconfiguration of radial distribution networks: application of heuristic methods on large-scale networks. IEEE Proceedings Generation, Transmission and Distribution, v. 142, n. 6, p. 631-638, 1995.

Pereira, Fernando Silva. Reconfiguração ótima de sistemas de distribuição de energia elétrica baseado no comportamento de colônias de formigas. 2010. Tese (Doutorado em Engenharia Elétrica) - Escola de Engenharia de São Carlos da Universidade de São Paulo, São Paulo, 2010.

Salume, Gustavo N. et al. Reconfiguração de sistemas de distribuição desbalanceados utilizando metodologia heurística e fluxo de potência trifásico. Anais do XVI Congresso Brasileiro de Automática, Salvador, p. 2263-2268, 2006.

Shirmohammadi, Dariush; Hong, H. Wayne. Reconfiguration of electric distribution network for resistive line losses reduction. IEEE Transactions On Power Delivery, v. 4, n. 2, p. 1492-1498, 1989.

$\mathrm{Su}$, Ching-Tzong; Lee, Chu-Sheng. Network reconfiguration of distribution systems using improved mixed-integer hybrid differential evolution. IEEE Transactions On Power Delivery, v. 18, n. 3, p. 1022-1027, 2003.

Sulaiman, Mohd Herwan et al. Optimal allocation and sizing of distributed generation in distribution system via firefly algorithm. Power Engineering and Optimization Conference (PEDCO) in Malaysia, 2012.

Yang, Xin-She. Nature-inspired metaheuristic algorithms. 2. ed. United Kingdom: Luniver Press, 2010.

\title{
RESPONSABILIDADE AUTORAL
}

“Os autores são os únicos responsáveis pelo conteúdo deste trabalho".

\section{HEURISTIC ALGORITHM FOR ELECTRICAL DISTRIBUTION SYSTEMS RECONFIGURATION BASED ON FIREFLY MOVEMENT EQUATION}

\author{
Lindenberg Isac da Silva, lindenberg.isac@ufabc.edu.br ${ }^{1}$ \\ Edmarcio Antonio Belati, edmarcio.belati@ufabc.edu.br² \\ Ivo Chaves da Silva Junior, ivo.junior@ufjf.edu.br ${ }^{3}$ \\ ${ }^{1,2}$ Federal University of ABC - UFABC - Engineering Center, Modeling and Applied Social Sciences \\ Abolição Street, s/nº - São Pedro Village - CEP 09210180 - Santo André - SP - Brazil \\ ${ }^{3}$ Federal University of Juiz de Fora - UFJF - Electrical Energy Department \\ José Lourenço Kelmer Street, s/nº - São Pedro - CEP 36036900 - Juiz de Fora - MG - Brazil
}

\begin{abstract}
A heuristic computational method based on the firefly movement equation is presented aiming at optimizing the electrical power distribution to the customers and minimizing the waste of ohmic losses due to Joule effect. The proposed algorithm simulates the fireflies movement towards preys or partners to match and, through a discretization, the insects positions in the space correspond to the positions of the switches in the electrical system. Using the suggested heuristic we can reduce the problem of exponential increase of the possible switches combinations to reconfigurate the electrical system. Four systems were used in the tests: sixteen bus and sixteen switches, sixteen bus and twenty-one switches, thirty-three bus and thirty-seven switches and seventy bus and seventy-four switches. The algorithm is able to detect and exclude net typologies that transgress operational restrictions such as the system radiality and the voltage profile maintenance.
\end{abstract}

